Particle Acceleration Very Near an X-Line in a Collisionless Plasma
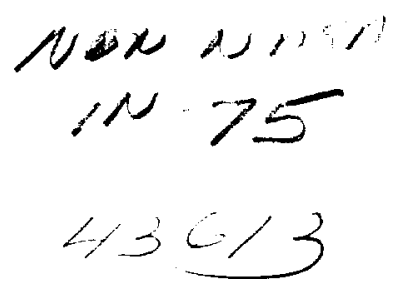

15 March 1995

Prepared by

L. R. LYONS and D. C. PRIDMORE-BROWN

Space and Environment Technology Center

Technology Operations

Prepared for

NASA/GODDARD SPACE FLIGHT CENTER

Greenbelt, MD 20771

Grant No. NAGW-2126

Engineering and Technology Group 
Aerospace Report No.

ATR-92(7251)-4

\title{
PARTICLE ACCELERATION VERY NEAR AN X-LINE IN A COLLISIONLESS PLASMA
}

\author{
Prepared by
}

L. R. Lyons and D. C. Pridmore-Brown Space and Environment Technology Center

Technology Operations

15 March 1995

Engineering and Technology Group THE AEROSPACE CORPORATION

El Segundo, CA 90245-4691

Prepared for

NASA/GODDARD SPACE FLIGHT CENTER

Greenbelt, MD 20771

Grant No. NAGW-2126

PUBLIC RELEASE IS AUTHORIZED 
Aerospace Report No.

ATR-92(7251)-4

PARTICLE ACCELERATION VERY NEAR AN X-LINE

IN A COLLISIONLESS PLASMA

Prepared

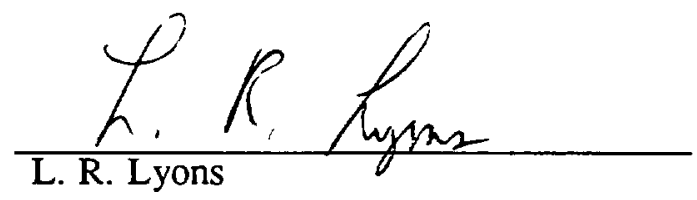

$$
\text { S. G: Predine- Premen }
$$

D. C. Pridmore-Brown

Approved
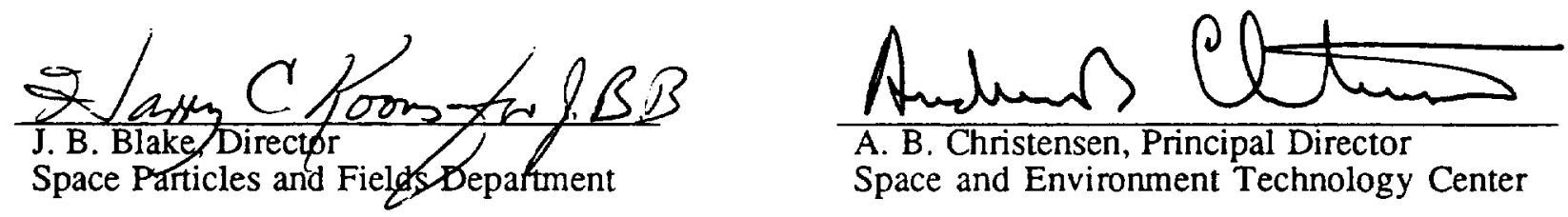


\section{NOTE}

The material reproduced in this report originally appeared in Geophysical Monograph 86, Space Plasmas: Coupling Between Small and Medium Scale Processes. The ATR is published to document the work for the corporate record. 


\title{
Particle Acceleration Very Near an X-Line in a Collisionless Plasma
}

\author{
L. R. LYONS AND D. C. PRIDMORE-BROWN
}

Space and Environment Technology Center. The Aerospace Corporation. El Segundo, Califormia 90245

\begin{abstract}
In a previous paper, we applied a simplified model for particle motion in the vicinity of a magnetic X-line that had been introduced by Dungey. We used the model to quantitatively show that an electric force along an $\mathrm{X}$-line can be balanced by the gyroviscous force associated with the off-diagonal elements of the pressure tensor. Distribution functions near the $\mathrm{X}$-line were shown to be skewed in azimuth about the magnetic field and to include particles accelerated to very high energies. In the present paper, we apply the previous model and use the distribution functions to evaluate the energization that results from particle interactions with the $\mathrm{X}$-line We find that, in general, this interaction gives a spectrum of energized particles that can be represented by a Maxwellian distribution. A power-law, high-energy tail does not develop. The thermal energy, $K$, of the Maxwellian can be expressed simply in terms of the field parameters and particle mass and charge. It is independent of the thermal energy, $K_{i}$, of the particle distribution incident upon the region of the $\mathrm{X}$-line, provided that $\mathrm{K}_{\mathrm{i}}<\mathrm{K}$. Significant energization is not found for $K_{i}>K$.
\end{abstract}

\section{INTRODUCTION}

Energy transfer in space plasmas is often associated with a magnetic X-line along which lies an electric field [e.g. Vasyliunas, 1975], and the physics of the forces and particle acceleration near such $\mathrm{X}$-lines is an active research topic. In this paper, we consider the acceleration of particles very near an X-line using particle distributions that are consistent with the requirement for force balance near the $\mathrm{X}$-line

Plasma dynamics near an X-line are often addressed within the framework of $\mathrm{MHD}$ under the assumption that some sort of resistivity maintains the force that opposes the electric force along the $\mathrm{X}$-line. However, space plasmas are generally collisionless so that the applicability of the concept of resistivity is highly questionable.

It has been pointed out [Vasyliunas, 1975; Sonnerup, 1979,1988 ] that force balance along an X-line in a collisionless plasma requires a balance between the electric force and the gradient of the off-diagonal elements of the electron pressure tensor (referred to as "gyroviscosity"); however,

\footnotetext{
Space Plasmas: Coupling Between Small

and Medium Scale Processes

Geophysical Monograph 86

Copyright 1995 by the American Geophysical Union
}

identification of a process that could lead to such gyroviscosity was not accomplished until recently. The breakthrough on this topic was by Dungey [1988], who evaluated collisionless particle motion near an X-line using a simplified model. He showed that the particle motion near an X-line will lead to a skewing of the velocity-space particle distributions and thus to significant off-diagonal pressure tensor elements. He suggested that the resulting gyroviscosity could be sufficient to balance the electric force along the $X$ line. Such gyroviscosity should be present in simulations of collisionless reconnection and should be identifiable by evaluation of the off-diagonal elements of the pressure tensor.

We [Lyons and Pridmore-Brown, 1990; hereafter referred to as paper 1] applied Dungey's model to evaluate particle distribution functions very near an X-line, and we used these distributions to quantitatively evaluate the force balance. We found that, within the limits imposed by Dungey's model, gyroviscosity can balance the electric force along an $X$-line in a collisionless plasma independent of other plasma and field parameters. The distribution functions we evaluated include the effects of particle energization from electric field acceleration in the weak magnetic field region near the $\mathrm{X}$-line. Here we use these distributions to evaluate this energization. 


\section{DUNGEYS MODEL}

The magnetic field (B) model used to evaluate the particle trajectories and distribution functions is two-dimensional, having $\partial / \partial y=B_{y}=0$. The $X$-line is taken to lie along the $y$ axis and to be imbedded within a current sheet that is centered in the $\mathrm{z}=0$ plane. $\mathrm{B}_{\mathrm{X}}$ reverses in sign across the current sheet. Symmetry is assumed about the $X$-line, so that $B_{X}(z)=-B_{X}(-z)$, and $B_{Z}(x)=-B_{Z}(-x)$. The normal component of $B$ across the current sheet is expanded in a Taylor series about the $X$-line, so that $B_{z}=\beta x$. The electric field $E_{y}$ is taken to be uniform and to be directed parallel to the current in the positive $y$-direction.

The basic motion of particles in the vicinity of a current sheet having a weak but uniform $B_{Z}$ was originally presented by Speiser [1965] and is described in paper 1. Particles that reach the midplane of the current sheet oscillate about the midplane and simultaneously gyrate about $\mathrm{B}_{\mathbf{Z}}$ for one-half a gyro-circle; the gyration begins with an $x$-component of velocity, $v_{x o}$, directed towards the $X$-line, and $v_{y o}=0$. This gives a current in the positive $y$-direction, as is required to maintain the current of the current sheet. Only particles with $\mathrm{gv}_{\mathrm{y}}>0$ remain in the current sheet. This is because particles with $\mathrm{qv}_{\mathrm{y}}<0$ are accelerated away from the current sheet by the magnetic force due to $B_{x}$ (which the electrons encounter as a result of their oscillatory motion about $z=$ 0).

Following Dungey [1988] and paper 1, we take advantage of the decoupling that occurs between the $x$-y component and the $z$ component of motion for particles as they oscillate about the $z=0$ plane [Speiser, 1965, 1968]. This decoupling is assumed to hold as the $x-y$ component of a particle trajectory crosses an $\mathrm{X}$-line. Speiser did not specifically include this situation or the effects of $\partial \mathrm{B}_{\mathrm{Z}} / \partial \mathrm{x} \neq 0$ in his analysis, though particle behavior near an $\mathrm{X}$-line has been considered by Stern [1979]. The evaluation of particle motion within the midplane of the current sheet can thus be simplified by calculating only the $x-y$ component of the particle trajectory at $z=0$. This motion is taken to begin with $v_{y}=0$.

Under the above conditions, the equations of motion for particles of charge $q$ and mass $m$ are:

$$
\begin{gathered}
m\left(d v_{x} / d t\right)=q \beta x v_{y} \\
m\left(d v_{y} / d t\right)=q\left(-\beta x v_{x}+E_{y}\right) .
\end{gathered}
$$

Using the normalized temporal and spatial variables introduced by Dungey [1988],

$$
\begin{aligned}
x^{\prime}=x / L, & \text { where } L=\left|E_{y} m /\left(\beta^{2} q\right)\right|^{1 / 3} \\
t^{\prime}=t / \tau, & \text { where } \tau=L^{2} \beta /\left|E_{y}\right|,
\end{aligned}
$$

the equations of motion become

$$
\begin{gathered}
d v_{x}^{\prime} / d t^{\prime}=x^{\prime} v_{y} \operatorname{sign}(q) \\
d v_{y}{ }^{\prime} / d t^{\prime}=\left(-x^{\prime} v_{x}{ }^{\prime} \pm 1\right) \operatorname{sign}(q),
\end{gathered}
$$

where \pm refers to the sign of $E_{y}$, which we take to be positive in the present work. Note that $E_{y}, m, \beta$, and $q$ all have a magnitude of unity in normalized units. Also, since the equations of motion are applicable to particles of any charge, we take $\operatorname{sign}(q)=+1$. Thus, our results are applicable to electrons and ions provided the sign of $\mathrm{v} y^{\prime}$ is changed for electrons.

Representative particle trajectories in the vicinity of the $X$-line at $x^{\prime}=0$, obtained from solving equations (5) and (6) using a Runge-Kutta scheme, are shown in Figure 1. The trajectories shown are for particles that start their motion within the current sheet at values of $x^{\prime} \geq 0$. The distortion of the trajectories from semicircles results from the acceleration by the electric field and the variation of $B_{Z}$ with $x^{\prime}$.

\section{VELOCITY SPACE DISTRIBUTIONS}

Trajectories such as shown in Figure 1 can be used to evaluate normalized distribution functions, $f$, in phase space for the $z^{\prime}=0$ plane if a distribution function, $f_{i}^{\prime}$, is specified for particles incident upon that plane. In paper 1, we illustrated the features of these distributions by taking a "top hat" form for $f_{i}^{\prime}$. The value of $f_{i}^{\prime}$ was taken to be a constant for all initial velocities, $v_{x 0}$ ', directed toward the $X$-line and having $0 \leq\left|v_{x 0^{\prime}}\right| \leq 1$, and $f_{i}^{\prime}$ was taken to be zero otherwise. Particles were assumed to be incident at all values of $x^{\prime}$. With this incident distribution, the distribution at all $x^{\prime}$ in the $z^{\prime}=0$ plane can be described by an area in $v_{x}^{\prime}, v^{\prime}$ space within which $f$ is constant and outside of which $f^{\prime}=0$. It thus suffices to trace the boundary of this area in order to describe $f\left(v_{x}^{\prime}, v_{y}\right)$ for an $x^{\prime}$ at $z^{\prime}=0$.

Boundaries in velocity space within which $f$ is constant, as obtained in paper 1 for selected values of $x^{\prime}$, are shown in Figure 2. The boundaries show that $f$ becomes increasingly skewed towards positive $v_{x^{\prime}}$ and $v_{y^{\prime}}$ as $x^{\prime} \rightarrow 1$. This is the skewing that gives rise to positive values for the offdiagonal elements of the pressure tensor and thus to the gyroviscosity. In paper 1 , we used these distributions to evaluate moments of $f$ and the balance of forces near the $X$ line.

While all particles used to construct the distributions in Figure 2 entered the $z^{\prime}=0$ plane with $\left|v^{\prime}\right| \leq 1$, the distributions show tails extending to very high values of $v^{\prime}$. These tails result from particles having trajectories that become nearly parallel to the $X$-line at very small values of $x^{\prime}$ (see trajectories in Figure 4 of paper 1), and the tails are associated with a net energization of particles that results from 


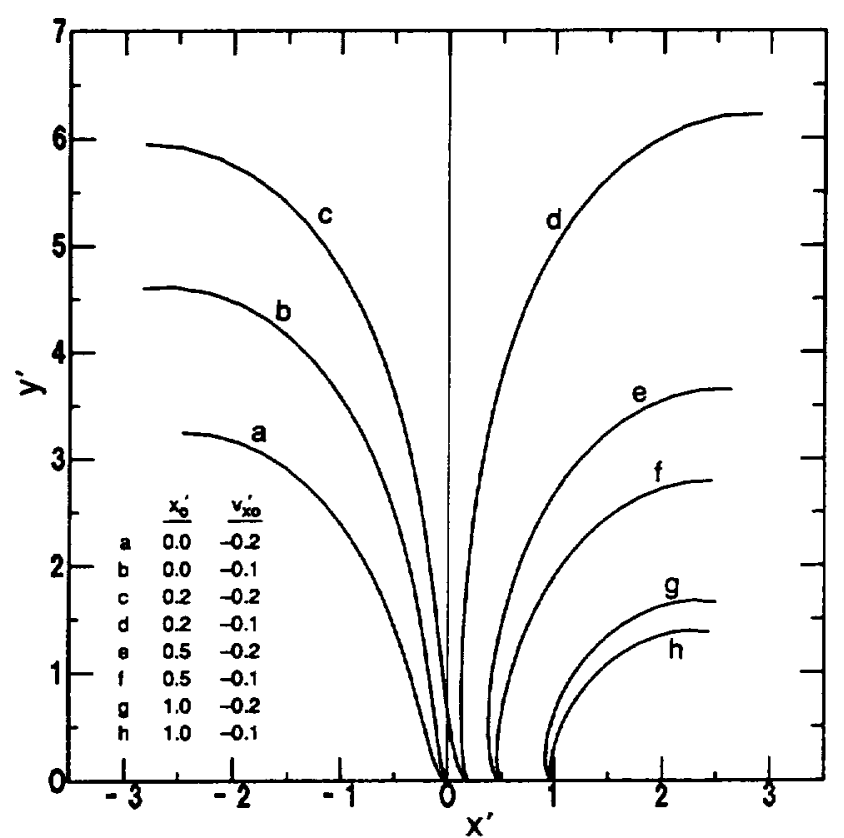

Fig. 1. Representative particle trajectories in the $x^{\prime}-y^{\prime}$ plane near an $X$-line at $x^{\prime}=0$. The sign of $y^{\prime}$ is correct for positively charged particles. For negatively charged particles, the sign of $y^{\prime}$ must be changed.

their interaction with the $\mathrm{X}$-line.

To evaluate the energization of particles from their interaction with the X-line, we assume a Maxwellian,

$$
F_{i}\left(v_{x o}\right)=\exp \left[-v_{x 0^{\prime}} / v_{i}{ }^{2}\right],
$$

for the distribution of initial speeds of particles following their incidence upon the $\mathrm{z}=0$ plane. Under the assumption that initial velocities are directed towards the $\mathrm{X}$-line, equation (7) corresponds to an incident distribution function in velocity space given by:

$$
\begin{aligned}
& f_{i}^{\prime}\left(v_{x 0^{\prime}}, v_{y o}{ }^{\prime}, v_{z o}\right)=\exp \left[-v_{x o^{\prime}}{ }^{2} / v_{i}{ }^{2}\right] \delta\left(v_{y o}\right) f_{i}^{\prime}\left(v_{z o}{ }^{\prime}\right) \text {, } \\
& \text { for } x^{\prime} v_{x o}{ }^{\prime} \leq 0 \text {, }
\end{aligned}
$$

where $\int f_{i^{\prime}}\left(v_{z O^{\prime}}\right) d v_{z 0^{\prime}}=1$, and $f_{i}{ }^{\prime}=0$ for $x v_{x 0^{\prime}}>0$. Applying the assumption that the $z$ and $x-y$ components of particle motion are decoupled within the $\mathrm{z}=0$ plane, we take If $\left(v_{z}\right) d v_{z}^{\prime}=1$ throughout the $z^{\prime}=0$ plane and ignore the $v_{z^{\prime}}$ dependence of $f$.

Using equations (3) and (4), the normalized thermal velocity of incident particles can be expressed in terms of real parameters as

$$
v_{i}^{\prime}=\left[2\left(\mathrm{~K}_{\mathrm{iq}}{ }^{1 / 3} \beta^{2 / 3}\right) /\left(\mathrm{E}_{\mathrm{y}}^{4 / 3} \mathrm{~m}^{1 / 3}\right)\right]^{1 / 2}
$$

where $\mathrm{K}_{\mathrm{i}}$ is the thermal energy of the distribution expressed in $\mathrm{eV}$. If we chose as nominal parameters for the distant geomagnetic tail $K_{i}=110 \mathrm{eV}, \beta=0.3 \mathrm{nT} / R_{e}$, and $E_{y}=$ $0.25 \mathrm{mV} / \mathrm{m}$, then equation (9) gives $v_{i}^{\prime}=1.0$ and 0.286 for electrons and protons, respectively.

With a Maxwellian distribution for $F_{i}^{\prime}\left(v_{x o}\right), f\left(v_{x}, v_{y}{ }^{\prime}\right)$ does not divide into regions where $f=$ constant and where $f=$ 0 . However, the regions of maximum $f$ remain similar in shape to the boundaries shown in Figure 2, so that the distributions in Figure 2 still qualitatively represent $f\left(v_{x}^{\prime}, v_{y}{ }^{\prime}\right)$. To quantitatively evaluate $f^{\prime}\left(v_{x^{\prime}}, v_{y}\right)$ at a given $x^{\prime}$, we use equations (5) and (6) to trace particle trajectories backwards in time from points in $v_{x}{ }^{\prime}, v_{y^{\prime}}$ space until $v_{y}{ }^{\prime}=0$. In this way, we find initial speeds, $v_{x 0^{\prime}}$, corresponding to the points in $v_{x}, v_{y}{ }^{\prime}$ space, and we obtain $f^{\prime}$ from equation (8).

Since our goal is to evaluate the energization from particle interactions with an X-line, we choose values of $v^{\prime}=$ $\left.\left(v_{x}{ }^{2}+v_{y}\right)^{2}\right)^{12}$ and determine $f$ over a range of values for $f$ $=\tan ^{-1}\left(v_{y} / v_{x}\right)$ selected so as to allow the evaluation of $F\left(v^{\prime}\right)=\int f^{\prime}\left(v^{\prime}, \phi\right) d \phi / \pi$. This gives the distribution of speeds for particles within the current sheet after their interaction with the X-line, which can be compared directly with the incident distribution given by equation (7). As can be seen from Figure 2, the range of important $\phi$ values becomes increasingly narrow with increasing $v^{\prime}$, and, in fact, becomes several orders of magnitude less than $1^{\circ}$ wide for the highest $v^{\prime}$ values of interest. This narrow range would be difficult to handle in a model that includes three-dimensions of particle motion; however, it does not present any problems bere.

Comparisons between $F$ and $F_{i}{ }^{\prime}$, plotted versus $v^{\prime} / v_{i}{ }^{\prime}$ for $x^{\prime}=1$, are displayed in Figure 3. In principal, the results are only dependent on $v_{i}^{\prime}$. However, we show results for values of $v_{i}$ ' that correspond to specific real parameters. Results for the values of $v_{i}^{\prime}$ given above that correspond to electrons and ions for nominal tail parameters are shown in the middle panel (identified as $\mathrm{E}_{\mathbf{y}}=0.25 \mathrm{mV} / \mathrm{m}$ ). Results are also shown for values of $v_{i}^{\prime}$ that have been adjusted, using equation (9), for $E_{y}=0.062 \mathrm{mV} / \mathrm{m}$ and for $E_{y}=1.0 \mathrm{mV} / \mathrm{m}$ with $K_{i}$ and $\beta$ unchanged. In each panel, the solid line gives $F_{i}$. When plotted versus $v^{\prime} / v_{i}{ }^{\prime}, F_{i}{ }^{\prime}$ is the same for all $v_{i}{ }^{\prime}$, which facilitates comparison of the amount of energization between the different cases. Our evaluation of $F(v)$ for each $E_{y}$ is given by filled circles for the value of $v_{i}$ corresponding to electrons and by filled triangles for the value of $v_{i}{ }^{\prime}$ corresponding to protons.

Figure 3 shows significant energization for all cases having $v_{i}^{\prime} \leq 1$, the energization increasing with decreasing $v_{i}^{\prime}$. Such energization does not occur for $v_{i}{ }^{2}$, 1 , as can be seen for electrons with $E_{y}=0.062 \mathrm{mV} / \mathrm{m}$, which corresponds to $v_{i}^{\prime}=2.5$. Keeping other parameters fixed, the energization is greater for protons than for electrons, since $v_{i}^{\prime} \propto \mathrm{m}^{-1 / 6}$, 

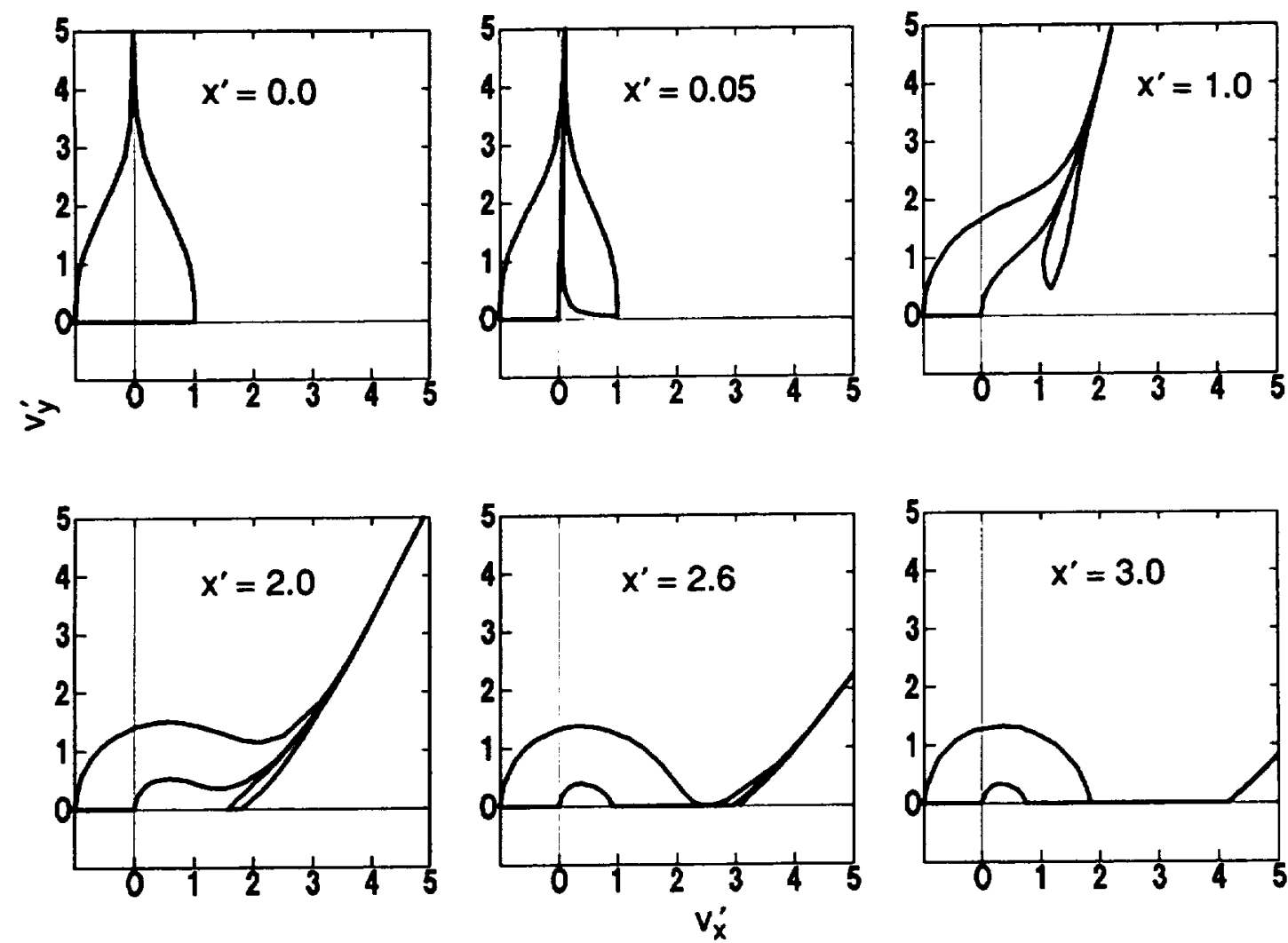

Fig. 2. Boundaries in normalized velocity space within which $f^{\prime}$ is constant at selected values of $x^{\prime}$ as obtained in paper 1 for the "top-hat" distribution of particles incident upon the current sheet.

and it increases with $E_{y}$ since $v_{i} \propto E_{y}-2 / 3$.

The distributions in Figure 2 suggest that the energy distributions for the particles might show a high energy tail since some low-energy particles are accelerated to very high energies. However, Figure 3 shows that $F^{\prime}\left(v^{\prime}\right)$ falls off as a Maxwellian for $v^{\prime}>v_{i}^{\prime}$. In fact, we have found that $F\left(v^{\prime}\right)$ at $v^{\prime} \geq 1$ is very well represented by a Maxwellian having a normalized thermal velocity equal to two for all the cases examined that have $v_{j}{ }^{\prime} \leq 1$. Specifically, the Maxwellian

$$
F\left(v^{\prime}\right)=0.5 v^{3 / 2} \exp \left[-v^{\prime} / 2\right]^{2}
$$

which is shown in Figure 3 by dashed lines, can be seen to fit our results very well for $v^{\prime} \geq 1$. All our distributions have $F^{\prime}\left(v^{\prime}\right) \rightarrow 0.5$ as $v^{\prime} \rightarrow 0$; thus equation (10) does not fit $F^{\prime}\left(v^{\prime}\right)$ for $v^{\prime} * 1$.

The results in Figure 3 are all for $x^{\prime}=1$. Results for the nominal tail parameters are shown in Figure 4 for $x^{\prime}=0,1$, and 2, which show that $F(v)$ is essentially independent of $x^{\prime}$ near the $\mathrm{X}$-line.
The results in Figures 3 and 4 show that the energy distribution of particles in a current sheet very near an X-line should have a thermal velocity of approximately two in normalized units, independent of the thermal energy of the particles incident upon the current sheet, provided the normalized thermal velocity of the incident particles $v_{i}{ }^{\prime} \leq 1$. This implies, from equations (3) and (4), that such particles will have a thermal energy $\mathrm{K}$ in $\mathrm{eV}$ given by

$$
K=2 E_{y}^{4 / 3} m^{1 / 3} / q^{1 / 3} \beta^{2 / 3},
$$

independent of $K_{i}$ provided $K \geq 4 K_{i}$. No energization occurs if equation (11) gives $K \leq \mathbf{K}_{\mathbf{i}}$. For our nominal tail parameters $\left(E_{y}=0.25 \mathrm{mV} / \mathrm{m}\right)$, equation (11) give a thermal energy of $0.4 \mathrm{keV}$ for electrons and $5.3 \mathrm{keV}$ for protons. These are of the order of the thermal energies of particles observed within the tail plasma sheet; however, the effects of particle motion away from the $\mathrm{X}$-line region would need to be evaluated for a direct comparison with plasma sheet observations to be meaningful. 


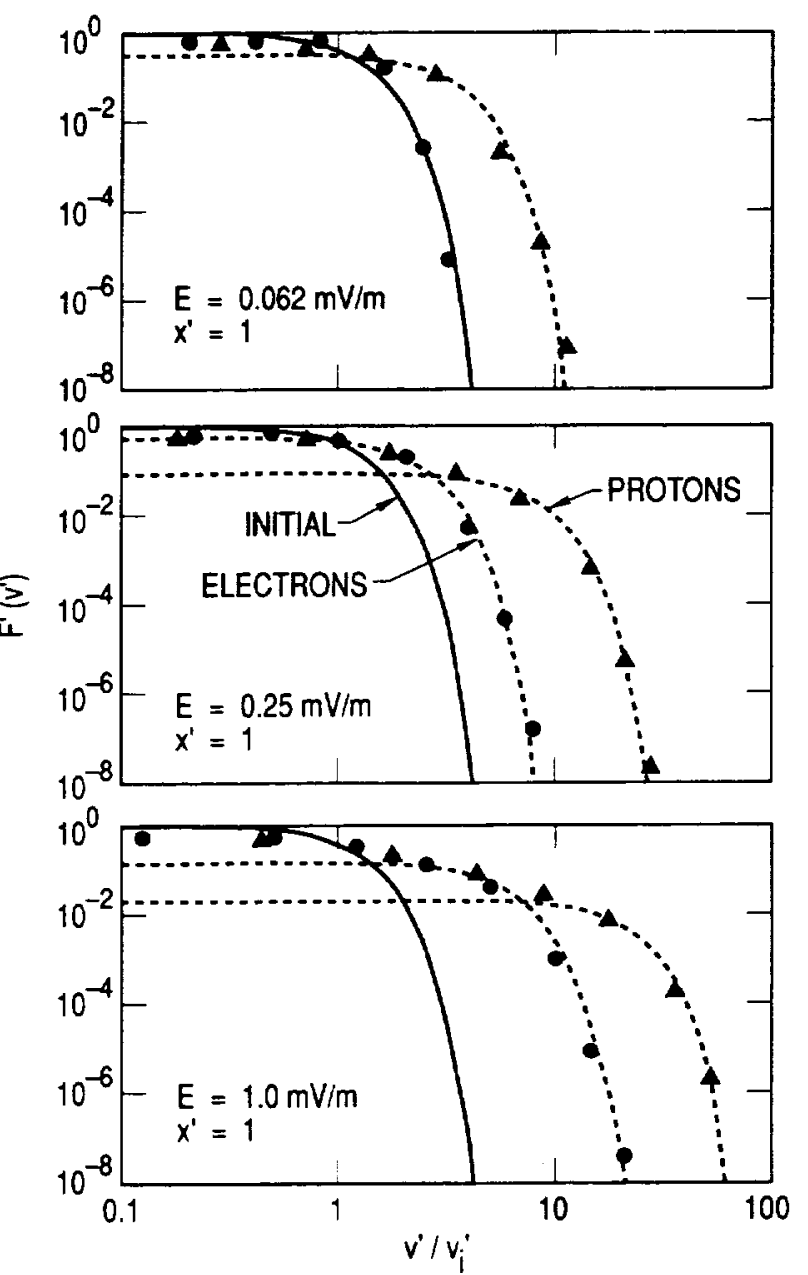

Fig. 3. Normalized distribution functions $F^{\prime}\left(v^{\prime}\right)$ versus $v^{\prime} / v_{i}^{\prime}$. In each panel, our evaluations of $F$ at $x^{\prime}=1$ are given by filled circles for electrons and by filled triangles for protons. The Maxwellian approximation for $F^{\prime}\left(v^{\prime}\right)$, equation (10), is given by the dashed lines, and the distribution of incident particles, equation (7), is given by the solid lines. The values of $v_{j}^{\prime}$ used for electrons and ions, respectively, are 2.50 and 0.715 in the upper panel, 1.00 and 0.286 in the middle panel, and 0.400 and 0.113 in the bottom panel. These values correspond to the electric fields given in each panel and $\beta=0.3 n T / R_{e}$.

\section{SPECTRA OF PARTICLES EJECTED FROM CURRENT SHEET}

The energy spectra calculated in the previous section would only be directly observable near the mid-plane of the tail current sheet and within a normalized distance $x^{\prime} \leq 2$ from an $\mathrm{X}$-line. This distance is quite small for electrons,
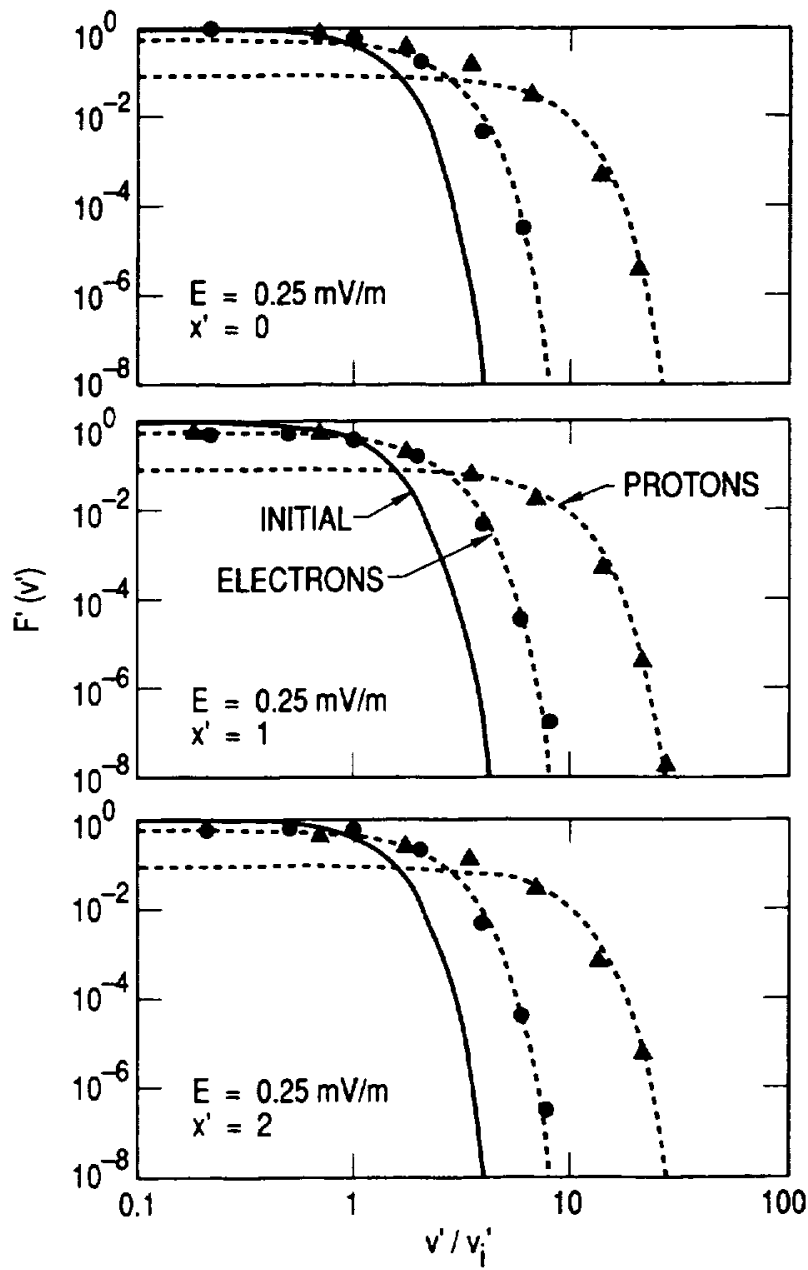

Fig. 4. Same as Figure 3, except that results are shown at $x^{\prime}=$ 0,1 , and 2 for the values of $v_{i}^{\prime}$ corresponding to the $0.25 \mathrm{mV} / \mathrm{m}$ electric field.

and is given by $2 \mathrm{~L}=1700 \mathrm{~km}$ for our nominal tail parameters. For protons, $2 \mathrm{~L}=20,800 \mathrm{~km}$. However, after traversing half a gyro-orbit about $\mathrm{B}_{\mathrm{Z}}$, all particles in our model are presumed to be ejected from the current sheet. These particles will flow along field lines just within the boundary between open and closed field lines, where they are more likely to be observed than within the current sheet. It thus is worthwhile to consider the energy spectra of the ejected particles.

Within the context of our model, it is not sufficient to simply evaluate the distribution function of ejected particles as a function of energy at a specified $x$. This is because of the strong variation of the location $x_{f}^{\prime}$ of particle ejection as 
a function of initial velocity $v_{x 0^{\prime}}$ and position $x_{0}{ }^{\prime}$. In particular, particles that attain high final velocities $v_{f}^{\prime}$ (i.e., $v_{f}^{\prime}$ $\geq 3$ ) are ejected over very narrow ranges of $x_{f}^{\prime}$ that become increasingly narrow, but occur at increasing $x^{\prime}$, with increasing $\mathbf{v}_{\mathbf{f}} \mathbf{f}^{\prime}$. For example, most particles with $\mathbf{v}_{f^{\prime}}=\mathbf{3}$ are ejected within a range -0.2 wide in $x^{\prime}$ centered at $x_{f}^{\prime}=2.6$, whereas particles with $v_{f}^{\prime}=6$ are primarily ejected within a range 0.0002 wide in $x^{\prime}$ centered at $x_{f}^{\prime}=3.536$. (These $x^{\prime}$ ranges have been determined using $\mathrm{f}_{\mathrm{i}}^{\prime}$ for $\mathrm{v}_{\mathrm{i}}^{\prime}=1$.) As a result, an ejection spectrum at a specified $x^{\prime}$ would consist mostly of particles having a very limited range of energies.

We thus have decided to evaluate the total number of ejected particles that have interacted with the region near the $X$-line as a function of $\mathbf{v}^{\prime}$. We do this by assuming that particles are incident upon the current sheet within a range of $x_{0}{ }^{\prime}$ centered about $x^{\prime}=0$, and evaluating $F_{T^{\prime}}\left(v_{f}{ }^{\prime}\right)=$ $\int f^{\prime}\left(v_{f^{\prime}}, x_{f^{\prime}}\right) d \mathbf{d x}_{f^{\prime}}$ for various values of $v_{f^{\prime}}$. This integration gives us an energy spectra that can be interpreted as the spectra averaged over the spatial extent of the particles' gyromotion near the $\mathrm{X}$-line. Here we take the range of incident particles to be $\left|x_{0}\right| \leq 2.25$. This particular range was chosen because 2.25 is the minimum $\left|x_{f}^{\prime}\right|$ for incident particles having a speed of zero, the speed at which $F^{\prime}\left(v^{\prime}\right)$ maximizes, and we thus obtain a continuous spatial distribution for these particles. The precise size of this range, however, is not important to our results.

Energization of particles to high final velocities maximizes very close to $x^{\prime}=0$, whereas these energized particles are ejected beyond $\left|x^{\prime}\right|=2$. Thus, at higher speeds (i.e. $v^{\prime}$ $\geq 2$ ), we expect the distribution of ejected particles $\mathrm{F}_{\mathrm{T}}{ }^{\prime}\left(v_{\mathrm{f}}{ }^{\prime}\right)$ to be similar to the distributions $F\left(v^{\prime}\right)$ within the current sheet at $x^{\prime} \leq 2$ that are shown in Figures 3 and 4 . Figure 5 shows this similarity for the values of $v_{i}{ }^{\prime}$ that correspond to our nominal tail parameters. In Figure $5, \mathrm{~F}_{\mathrm{T}^{\prime}}\left(\mathrm{v}_{\mathrm{f}} \mathrm{f}^{\prime}\right)$ is given by open circles for electrons and by open triangles for protons. The similarity between $F_{T}^{\prime}\left(v_{f}^{\prime}\right)$ and $F^{\prime}\left(v^{\prime}\right)$ is also found for the other cases shown in Figure 3. (All particles are ejected with $v_{f^{\prime}} \geq 1$, so that $F_{T}^{\prime}\left(v_{f^{\prime}}\right)$ goes to zero at low values of $v_{f}^{\prime}$.) Thus, the energy spectra given by equation (10) is appropriate both within the current sheet and for the particles ejected from the X-line region. Again, however, an actual comparison with observations will require additional considerations of particle motion. In particular, the effects of particle mirroring and the resulting multiple interactions with the current sheet need to be evaluated.

\section{COMPARISON WITH PREVIOUS RESULTS}

Several studies have been performed that include the full three-dimensions of particle motion near an X-line. In this section, we compare our results to results from these studies. The comparisons provide a test of the assumptions

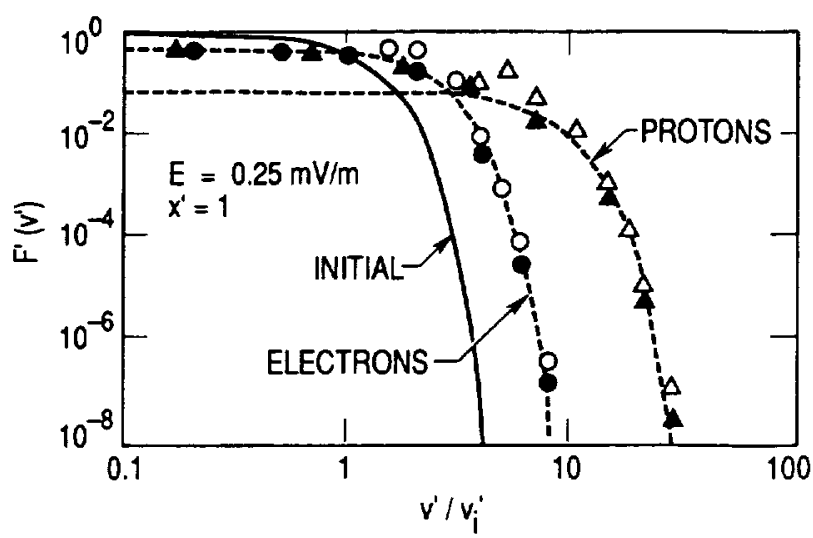

Fig. 5. Same as the middle panel of Figure 3, except the calculated distribution $F_{T}{ }^{\prime}\left(v_{f}{ }^{\prime}\right)$ of particles ejected from the current sheet has been added. Values of the ejected distribution are given by open circles for electrons and by open triangles for protons.

applied in our model to limit the evaluation of particle motion to two-dimensions. When three dimensions of motion are included, it becomes difficult to evaluate the trajectories of a sufficient number of particles to determine the spectra of energized particles to high energies, where the value of $F(v)$ is many orders of magnitudes below its peak near $v=0$. To the best of our knowledge, such a study has not been carried out. Also, none of the three-dimensional studies have considered the possibility of significant azimuthal asymmetries about $B$, which lead to the gyroviscosity and are crucial to the physics near the $X$-line. Nevertheless, a number of interesting results have been obtained that are relevant to the present study.

Martin and Speiser [1988] evaluated particle distributions that have been averaged over the azimuthal angle around $\mathbf{B}$. They found a ridge of enhanced values of $f$ in velocity space that results from particle energization near the X-line in their model. This ridge is probably the same feature as the tail extending to high energies in Figure 2; however, the skewing that gives rise to the gyroviscosity cannot be determined from their azimuthally averaged distributions.

Burkhart et al. [1990] considered cold particle distributions incident upon the region near an $\mathrm{X}$-line. They found that the velocity of the particle distribution flowing away from the X-line was proportional to $E_{y} 2 / 3 q^{1 / 3} / m^{1 / 3} \beta^{1 / 3}$, which is the same scaling relation that we have obtained [equation (11)] for the thermal velocity. They found that the outflowing particles were magnetic field-aligned. This information cannot be obtained from our evaluation of particle motion. However, if we assume that particles ejected from the current sheet in our model are field aligned, then we 
obtain the same scaling as did Burkhart et al. for the outflow velocity. Thus, their results provide an excellent verification of the scaling that we have obtained.

Deeg et al. [1991] followed the three-dimensional trajectories of 14,000 cold ( 1 eV thermal energy) protons near an $\mathrm{X}$-line and were able to obtain a statistically significant energy spectrum of energized particles for one set of conditions. They assumed that particles are incident upon the $X$ line over a limited range of $x$ near the $X$-line, and they counted the total number of particles ejected from the $\mathrm{X}$-line region as a function of energy. This procedure is essentially the same as ours, so that their spectrum provides an excellent test for our two-dimensional results. Their spectrum is shown in Figure 6 as the total number of particles per unit energy $N(K) d K$ [which is proportional to ${ }^{2} F_{T}(v)$ ] versus particle energy.

They used $E_{y}=0.3 \mathrm{mV} / \mathrm{m}$ and $\beta=2.5 \times 10^{-15} \mathrm{~T} / \mathrm{m}$. From equation (11), our analysis predicts a Maxwellian distribution of energized particles having a thermal energy of $480 \mathrm{eV}$. The dashed line in Figure 6 shows a Maxwellian distribution having a thermal energy of $300 \mathrm{eV}$ (which provided a better fit then did $480 \mathrm{eV}$ ), which can be seen to fit the simulated data very well. This comparison shows very good agreement between the general results from our twodimensional model and the three-dimensional results for the particular set of parameters used by Deeg et al. [1991], the difference in thermal energies being not very significant. Also, using 500 particles, Deeg et al. investigated how the energization varies with $E_{y}$ and $\beta$, and they found the same variation that we have found.

Note that even with 14,000 particles, Deeg et al.'s [1991] spectrum does not extend to high enough energies to distinguish a power-law high-energy tail from a Maxwellian. Our spectra do show this distinction, whereas their spectrum at energies above $\sim 0.5 \mathrm{keV}$ can be fitted as well by a power law as by a Maxwellian. However, the excellent agreement we find with their results gives strong support to the validity of our general two-dimensional result for the particle energization.

Goldstein et al. [1986] and Ambrosiano et al. [1988] evaluated the acceleration near X-lines and included the effects of magnetic turbulence obtained from MHD simulations. They found significant acceleration due to particle trapping in magnetic bubbles that developed in the simulations. Our results, however, are not directly comparable to their results because we did not allow for turbulence and because the basic physics of collisionless particle motion near an X-line (e.g., the gyroviscous force and the associated asymmetries in particle distribution functions about $B$ ) does not exist in MHD.

Finally, it is interesting to compare the results for parti-

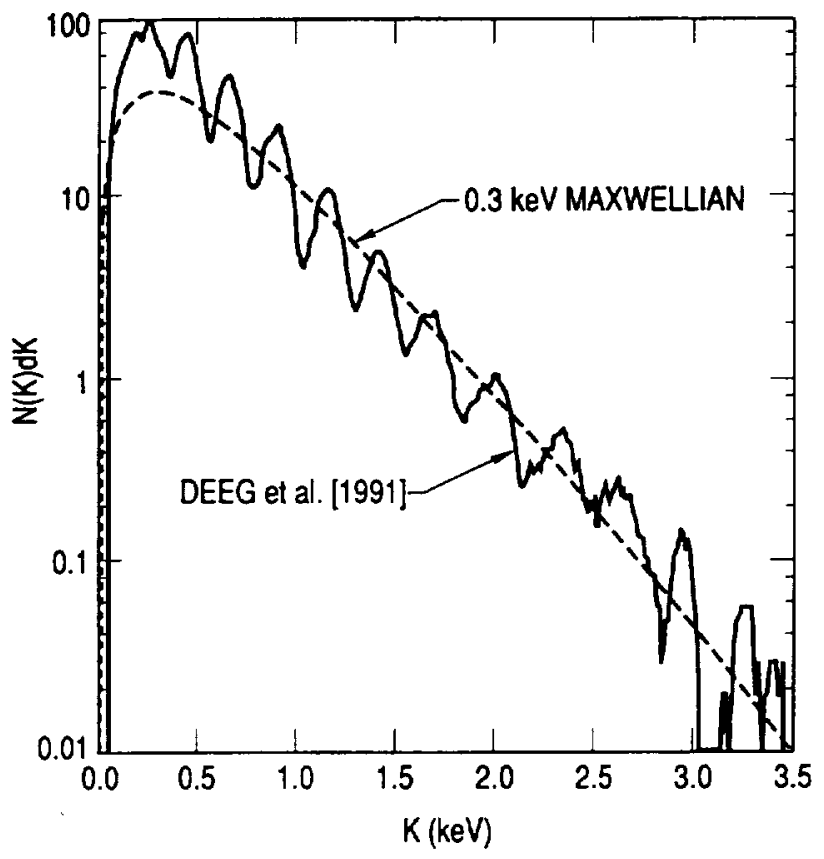

Fig. 6. Total number of particles per unit energy ejected from the region near an X-line as calculated by Deeg et al. [1991] from a three-dimensional simulation of particle motion. The dashed line gives a Maxwellian distribution for $0.3 \mathrm{keV}$ thermal energy.

cle energization near an $\mathrm{X}$-line with those obtained by Speiser and Lyons [1984] for a weak, but constant, $B_{Z}$ across a current sheet. They found that initially cold particles were accelerated to a final velocity $v_{f}=2 E_{y} / B_{z}$. Our present results give a thermal velocity $v_{t h}=$ $2 E_{y} 2 / 3 q^{1 / 3} / m^{1 / 3} \beta^{1 / 3}=2 E_{y} / B_{z}\left(x^{\prime}=1\right)$. Thus the present results give a thermal velocity that is the same as the final velocity in the $B_{Z}$ constant model, if $B_{Z}$ is evaluated one scale length $\mathrm{L}$ away from the $\mathrm{X}$-line.

\section{CONCLUSIONS}

We have applied Dungey's [1988] two-dimensional model of fields and collisionless particle motion to evaluate the particle energization near a magnetic $X$-line. We find that this process results in an energy spectrum of particles that can be approximated as a Maxwellian. The same Maxwellian applies for both the particles within the current sheet in the vicinity of the $\mathrm{X}$-line and for the particles ejected from that region of the current sheet. This Maxwellian has a thermal energy, $K$, given by equation (11), that depends upon field parameters but is independent of the thermal energy, $K_{i}$, of the particles incident upon the 
region of the $\mathrm{X}$-line. It is necessary that equation (11) give $K \geq K_{i}$ for interactions with an $X$-line to cause energization. No energization occurs if equation (11) gives $\mathrm{K}<\mathrm{K}_{\mathrm{i}}$, and (11) is not applicable to such a situation.

For typical magnetotail parameters, the thermal energy given by equation (11) is a few hundred $\mathrm{eV}$ for electrons and a few keV for protons. These energies are of the order of magnitude of those observed in the distant tail plasma sheet, which suggests that interactions with the neutral line in the distant tail may contribute to the energization of plasma sheet particles. During gecomagnetic disturbances, induction by time-varying magnetic fields may yield electric fields in the tail that are significantly enhanced over typical values. Taking $E_{y}=4 \mathrm{mV} / \mathrm{m}$ as perhaps an extreme example, we obtain thermal energies of $17 \mathrm{keV}$ for electrons and $210 \mathrm{keV}$ for protons. This is consistent with the suggestion that interactions with a neutral line may contribute to energetic particle phenomena that have been observed in the tail during substorms [e.g., Armstrong and Krimigis , 1968; Krimigis and Sarris, 1979; Baker at al., 1979].

We have compared our results to results from threedimensional studies of particle motion near an $\mathrm{X}$-line. These comparisons show our results to be consistent with the parameter scalings and the one detailed energy spectra obtained from the three-dimensional studies. Our results compliment the three-dimensional studies by giving an accurate energy spectrum to high energies that can be scaled to different magnetic and electric field parameters, and by providing details of the particle distribution functions such as the azimuthal asymmetries very near an $\mathrm{X}$-line.

Acknowledgements. We are grateful to J. W. Dungey for stimulating discussions that motivated this study and to $M$. Schulz for valuable discussions throughout the course of the study. This work was supported by the NASA Space Physics Theory Program grant NAGW-2126.

\section{REFERENCES}

Ambrosiano, J., W. H. Matthaeus, M. L. Goldstein, and D. Plante, Test particle acceleration in turbulent reconnecting magnetic Fields, J. Geophys. Res., 93, 14,383, 1988.

Armstrong, T. P., and S. M. Krimigis, Observations of protons in the magnetosphere and magnetotail with Explorer 33, J.
Geophys. Res., 73, 143, 1968.

Baker, D. N., R. D. Belian, P. R. Higbie, and E. W. Hones, Jr., High-energy magnetospheric protons and their dependence on geomagnetic and interplanetary conditions, J. Geophys. Res., 84, 7138, 1979.

Burkhart, G. R., J. F. Drake, J. Chen, Magnetic Reconnection in collisionless plasmas: Prescribed fields, J. Geophys. Res., 95, 18,833, 1990.

Deeg, H.-J., J. E. Borovsky, and N. Duric, Particle acceleration near X-type magnetic neutral lines, Phys. Fluids B, 3, 2660, 1991

Dungey, J. W., Noise-free neutral sheets, in Proceedings of an International Workshop in Space Plasma, held in Potsdam. GDR, ESA SP-285, Vol. II, p. 15, 1988.

Goldstein, M. L., W. H. Matthaeus, and J. J. Ambrosiano, Acceleration of charged particles in magnetic reconnection: solar flares, the magnetosphere, and solar wind, Geophys. Res. Lett., 13, 205, 1986.

Krimigis, S. M., and E. T. Sarris, Energetic particle bursts in the Earth's magnetotail, in Dynamics of the Magnetosphere, ed. S.-I. Akasofu, p. 599, D. Reidel, Dordrecht, Holland, 1979.

Lyons, L. R., and D. C. Pridmore-Brown, Force balance near an $X$-line in a collisionless plasma, J. Geophys. Res., 95, 20,903, 1990.

Martin, R. F., Jr., and T. W. Speiser, A predicted energetic ion signature of a neutral line in the geomagnetic tail, $J$. Geophys. Res., 93, 11521, 1988.

Sonnerup, B. U. O., Magnetic field reconnection, in Solar System Plasma Physics $I I I$, eds. L. J. Lanzerotti, C. F Kennel, and E. N. Parker, North-Holland, Amsterdam, p. 45, 1979.

Sonnerup, B. U. O., On the theory of steady-state reconnection, Computer Physics Communications, 49, 143, 1988.

Speiser, T. W., Particle trajectories in model current sheets, 1 , Analytical solutions, J. Geophys. Res., 70, 4219, 1965.

Speiser, T. W., and L. R. Lyons, Comparison of an analytical approximation for particle motion in a current sheet with precise numerical calculations, J. Geophys. Res., 89, 2177, 1984.

Stern, D. P., The role of O-type neutral lines in magnetic merging during substorms and solar flares, J. Geophys. Res., 84, 63, 1979.

Vasyliunas, V. M., Theoretical models of magnetic field line merging, 1, Rev. Geophys. Space Phys., 13, 303, 1975.

L. R. Lyons and D. C. Pridmore-Brown, The Aerospace Corporation, P. O. Box 92597, M2-260, Los Angeles, CA 90009 . 